\title{
Effective and quick discharge of residual fragments after minimal invasive stone procedures with "EPVL" modality: a new and promising approach
}

\author{
Kemal Sarica ${ }^{1}$
}

Received: 28 February 2020 / Accepted: 17 March 2020 / Published online: 2 April 2020

(c) Springer-Verlag GmbH Germany, part of Springer Nature 2020

Residual stone fragments and/or gravel following extracorporeal shock wave lithotripsy (SWL) or retrograde intrarenal surgery (RIRS) constitute a real challenge to the endourologists in clinical practice due to the high risk of stone regrowth, infection, obstruction as well as new stone formation. Although these fragments may pass spontaneously with conservative measures in a considerable percentage of the cases; this process may affect the patients's quality of life with the possible obstruction and distressing colic pain in most cases. Keeping this fact in mind, several noninvasive therapeutic strategies, including mechanical percussion, forced diuresis, body inversion (PDI), ultrasound waves and prototype magnetic tool have been introduced and applied in an attempt to facilitate the passage of these residual fragments after above mentioned minimal invasive stone management procedures. However, clinical efficacy of those measures remains really controversial [1].

Regarding this issue, researchers in China have worked on and designed a novel non-invasive device to promote active fragment discharge from the urinary system [2]. The main principle of this newly designed "External Physical Vibration Lithecbole (EPVL)" system is to ease the separation of the stone fragments (residing after endourological procedures) from the renal collecting system by exerting simple harmonic motion to the case on the table. In addition to the movement of the cases, a multidirectional harmonic vibration wave producing handle was used simultaneously to push the separated fragments forward out of the renal collecting system. Thus, through the combination of active positional change (in different directions) with an extracorporeal physical vibration to the involved lumbar area of the cases, an active and effective discharge of the stone fragments residing

Kemal Sarica

saricakemal@gmail.com

1 Department of Urology, Biruni University, Medical School, Medicana Bahcelievler Hospital, Istanbul, Turkey in different but particularly lower calyceal part of the treated kidney was aimed.

It is very clear and concise that for any newly designed and introduced technique to be well accepted, it should be first safe and also efficacious. Related with this issue, data obtained from several clinical studies have proved well with high-level evidence that the EPVL is a safe, simple, effective, and non-invasive method to facilitate the spontaneous passage of the residual fragments detected after RIRS or ESWL [2-6]. Of the published data with this system so far, Wu et al. [2] were able to demonstrate that EVPL as a supplement to RIRS could be more effective than RIRS alone by shortening the time period for stone clearance, increasing complete stone-free rates with good patient compliance and acceptance. Further supporting these findings, Zhang et al. [5] were able to show that the best time to perform EPVL is 3 days after RIRS procedure for the highest stone-free rates at any point in time with meaningfully reduced complications caused by these fragments. Last but not least, in their original studies, Long et al. [3], Wu et al. [6], and Tao et al. [4], all reported well that EPVL could facilitate the spontaneous discharge of residual stone fragments with a meaningfully shortened expulsion time after SWL. Thus all these data clearly indicate that the EPVL system can be regarded as an adjunctive active treatment modality particularly for residual stone fragments after RIRS and SWL procedures in an outpatient service manner.

Despite its first description a few years ago, EPVL system has yet to gain widespread popularity and acceptance by the endourologists. This modality will certainly prove itself to be as a new "adjunctive treatment" option to manage the residual stone fragments in cases undergoing above mentioned commonly performed procedures. Initial results are promising as well as encouraging enough to offer this procedure as a valuable alternative to the other conservative measures applied so far to obtain higher stone-free rates and also limit or avoid associated complications. In the light of 
the data published so far, I believe that EPVL will play a certain role in the quick and effective elimination of the residual stone fragments after RIRS and ESWL procedures which currently constitute a "real headache" for the majority of not only for the patients but also the endourologists themselves.

\section{Funding None.}

\section{Compliance with ethical standards}

Conflict of interest Prof.Kemal SARICA is acting as a consultant for Dornier GmbH company.

Ethical approval This article does not contain any studies with human participants performed by any of the authors.

\section{References}

1. Chiong E, Tay SPH, Li MK, Shen L, Kamaraj R, Esuvaranathan K (2005) Randomized controlled study of mechanical percussion, diuresis, and inversion therapy to assist passage of lower pole renal calculi after shock wave lithotripsy. Urology 65:1070-1074
2. Wu W, Yang Z, Xu C, Gu X, Yang S, Liao S, Wang R, Gao W, Ye Z, Zeng G (2017) External physical vibration lithecbole promotes the clearance of upper urinary stones after retrograde intrarenal surgery: a prospective, multicenter, randomized controlled trial. J Urol 197:1289-1295

3. Long Q, Zhang J, Xu Z, Zhu Y, Liu L, Wang H, Guo J, Wang G (2016) A Prospective randomized controlled trial of the efficacy of external physical vibration lithecbole after extracorporeal shock wave lithotripsy for a lower pole renal stone less than $2 \mathrm{~cm}$. J Urol 195:965-970

4. Tao R, Tang Q, Zhou S, Jia C, Lv J (2020) External physical vibration lithecbole facilitating the expulsion of upper ureteric stones $1.0-2.0 \mathrm{~cm}$ after extracorporeal shock wave lithotripsy: a prospective randomized trial. Urolithiasis 48:71-77

5. Zhang Y, Xu C, Wang Y et al (2019) When is the best time to perform external physical vibration lithecbole (EPVL) after retrograde intrarenal surgery (RIRS): a multi-center study based on randomized controlled trials. Urolithiasis. https://doi.org/10.1007/ s00240-019-01175-5

6. Wu W, Yang Z, Tang F et al (2018) How to accelerate the upper urinary stone discharge after extracorporeal shockwave lithotripsy (ESWL) for \%3c $15 \mathrm{~mm}$ upper urinary stones: a prospective multicenter randomized controlled trial about external physical vibration lithecbole (EPVL). World J Urol 36:293-298

Publisher's Note Springer Nature remains neutral with regard to jurisdictional claims in published maps and institutional affiliations. 